\title{
Safe and Minimally Invasive Laminoplastic Laminotomy Using an Ultrasonic Bone Curette for Spinal Surgery: Technical note
}

Kiyoshi Ito, M.D., Shigetoshi Ishizaka, M.D., Tetsuo Sasaki, M.D., Takahiro Miyahara, M.D., Tetsuyoshi Horiuchi, M.D., Keiichi Sakai, M.D., Hiroaki Shigeta, M.D*., and Kazuhiro Hongo, M.D.

Department of Neurosurgery, Shinshu University School of Medicine, Matsumoto 390-8621, *Department of Neurosurgery, Nagano Children's Hospital, Azumino 399-8288, Japan

Correspondence to:

Kiyoshi Ito, M.D.

Department of Neurosurgery,

Shinshu University School of Medicine, 3-1-1 Asahi, Matsumoto 390-8621, Japan

Telephone: 81-263-37-2690 Fax: 81-263-37-0480

E-mail: kitoh@shinshu-u.ac.jp

Running head: Ultrasonic bone curette for laminoplastic laminotomy 


\begin{abstract}
Background: Ultrasonic surgical aspirators have been used mainly for removing brain tumors. Because of their longitudinal and torsional tip, they are used for cutting the bone structures in spinal surgery installing a scalpel-type tip. The purpose of this report is to describe effectiveness and surgical pitfalls of an ultrasonic bone curette in laminoplastic laminotomy and hemilaminotomy.

Methods: The authors present 12 patients who underwent laminoplastic laminotomy and hemilaminotomy. We used a SONOPET UST-2001 ${ }^{\circledR}$ ultrasonic bone curette with HB-05S hand pieces (M\&M Co., Ltd., Tokyo, Japan). After a tumor was removed, titanium plates were used for the laminoplastic laminotomy and hemilaminotomy. The technical advantage of an ultrasonic bone curette and procedure-related complication were evaluated.
\end{abstract}

Results: There were no major procedure-related complications such as cord injury. Wound infection and subcutaneous fluid collection caused by cerebrospinal fluid leakage did not occur for reconstruction of posterior bony structure. In one patient with calcified dura mater associated with a tumor, dural tear occurred. The width of the tip was narrow enough for resected laminae to be fused postoperatively and spinal instability did not occur in all cases.

Conclusion: The scalpel-type ultrasonic bone curette is useful for cutting bone and effective for reconstruction of the laminae. Laminotomy with an ultrasonic bone curette is safe and minimally invasive. To prevent dural tear, we recommend drilling laminae to make the bone thin as the first step, followed by cutting the remaining laminae using a bone curette especially in cases with calcified or tense dura mater.

Key words: laminoplastic laminotomy, less invasive surgery, spinal cord tumor, ultrasonic bone curette 


\section{Introduction}

Laminoplastic laminotomy has been applied for the posterior approach for removal of spinal cord tumors. In this procedure, the resected lamina is returned after tumor removal, and laminoplasty is performed with titanium plates. This procedure allows stronger posterior support compared to laminectomy, preventing postoperative subcutaneous fluid collection caused by cerebrospinal fluid leakage and kyphotic deformity and infection caused by subcutaneous fluid collection ${ }^{4,6,8,9,11,18)}$. In addition, laminoplasty is thought to prevent recurrent canal stenosis by avoiding scar formation occurred by performing wide laminectomy, and this procedure reduces postoperative wound pain such as axial pain ${ }^{16)}$.

Conventional laminoplastic laminotomy, however, has a high technical demand on using high-speed drills, which may cause spinal cord and nerve injury with an inappropriate handling15). Spinal cord and root injury have been reported in laminotomy using a threadwire saw ${ }^{5}$. On the other hand, a scalpel-type ultrasonic bone curette, which has recently been introduced in the neurosurgical field, is an instrument for resecting bones with oscillation without rolling movement $2,3,13,17)$. It is commonly used for the intracranial surgery. Because of its longitudinal and torsional oscillating motions, it facilitates bone resection during spinal surgery ${ }^{7,14)}$. Scalpel-type ultrasonic bone curette, which was reformed, made it possible to cut the bony structure.

We performed laminoplastic laminotomy and hemilaminotomy for removal of the spinal cord tumor using the scalpel-type ultrasonic bone curette. We report our experience with the laminoplastic laminotomy and hemilaminotomy using the scalpel-type ultrasonic bone curette and discuss the technical advantages and safe usage of this instrument.

\section{Material and Methods}

The subjects were 12 patients who underwent laminoplastic laminotomy and hemilaminotomy between June 2006 and September 2007: 8 patients with neurinomas, one with cavernous angioma, perimedullary arteriovenous fistula of the cervical cord and two patients with tethered cord syndrome associated with a filar-type spinal lipoma. We used a SONOPET UST-2001 ${ }^{\circledR}$ scalpel-type ultrasonic bone curette with HB-05S hand pieces (M\&M Co., Ltd., Tokyo, Japan) for cutting the posterior laminae (Fig. 1 upper). The tips (0.7 $\mathrm{mm}$ wide) worked with scratching motions to resect bones (Fig. 1 lower). While the curette being operated, the hand piece end was cooled by automatic irrigation of physiological saline. Surgical procedures of laminoplastic hemilaminotomy were briefly described as follows: After a midline skin incision, unilateral paravertebral fascia and muscles were dissected to preserve the posterior tension band including the nuchal, supraspinous and interspinous ligaments and the spinous processes. The laminae were cut with a scalpel-type ultrasonic bone curette at the lamino-articular line and the base of the spinous process under an operating 
microscope (Fig. 2A). After exposure of dural theca, the intradural procedure was performed. After the dura mater was sutured, fixation with titanium miniplates (BEAR Medic Corporation, Tokyo, Japan) was carried out (Fig. 2B). A local bone which was harvested from the part of the spinous process was placed around the resected site. After performing laminoplasty, the paravertebral fascia, the subcutaneous tissue and the skin were sutured. For laminoplastic laminotomy, after bilateral paravertebral fascia and muscles were dissected, laminoplastic laminotomy were done using a scalpel-type ultrasonic bone curette and titanium miniplates. Schematic drawings of laminoplastic hemilaminotomy using a scalpel-type ultrasonic bone curette and titanium miniplates were described on Figure 3.

After the operation, the patient was instructed to wear a simple cervical collar or a lumbar brace for 3 weeks if cervical or lumbar laminoplastic laminotomy and hemilaminotomy was performed. No brace was required if a patient received thoracic laminoplastic laminotomy and hemilaminotomy.

\section{Results}

We experienced laminoplastic laminotomy and hemilaminotomy using a scalpel-type ultrasonic bone curette in 12 patients. Laminoplastic laminotomy and hemilaminotomy were safely and adequately accomplished with an ultrasonic bone curette. There were no major procedure-related intraoperative complications such as cord and root injuries. There was one patient who experienced dural tear without spinal cord injury. This case was a large dumbbell-shaped neurinoma with calcified dura mater, which was located at L1 to 3 level. After suturing the dura mater and covering it with NEOVEIL ${ }^{\circledR}$ (Gunze Limited, Tokyo, Japan) and fibrin glue, resected laminae were fixed to reconstruct the posterior bony structure.

Postoperative cerebrospinal fluid leakage did not occur.

All patients with at least a 6 -month follow-up had a tendency of fusion of the resected laminae on the computed tomographic (CT) scan (Table $1)$.

Postoperative spinal instability, cerebrospinal fluid leakage, wound infection and neurological deterioration did not occur in all patients.

\section{Illustrative cases}

Case 4: A 75-year-old woman complained of sensory disturbance of the bilateral upper extremities in 2000. The etiology was unclear despite detailed examination at a local clinic, and the patient was followed up. In 2006, sensory disturbance and motor weakness gradually extended to the lower extremities. A cervical magnetic resonance (MR) imaging revealed a tumor in the C7/Th1 (Fig. 4A). In January 2007, the tumor was removed with hemilaminotomy using the scalpel-type ultrasonic bone curette. The tumor originated from the posterior root of $\mathrm{C} 8$. After the tumor was totally removed, a bone flap was fixed to the lamina using titanium plates. There 
were no perioperative complications. Preoperative sensory disturbance of the bilateral upper extremities and dyskinesia of the right lower extremities relieved. The postoperative cervical radiographs and CT scan showed sufficient reconstruction of the spinal canal (Fig. 4B). A CT scan of the thoracic spine taken 10 months after operation demonstrated bony fusion of the lamina (Fig. 4C).

Case 7: A 38-year-old woman was admitted with a 4-year history of dysuria and weakness of the left lower extremity in 2007. Both MR images and CT scans of the lumbar spine revealed a large dumbbell-shaped tumor with calcified dura mater at L1 to 3 level (Fig. 5). In April 2007, the tumor was removed with laminotomy using the scalpel-type ultrasonic bone curette. During cutting the laminae, dural tear occurred. After suturing the dura mater and covering it with NEOVEIL ${ }^{\circledR}$ (Gunze Limited, Tokyo, Japan) and fibrin glue, resected laminae were fixed to reconstruct the posterior bony structure. There were no spinal cord injury and postoperative cerebrospinal fluid leakage. A CT scan of the lumbar spine taken 11 months after operation demonstrated bony fusion of the lamina (Fig. 5F).

\section{Discussion}

For removal of the spinal tumors, laminectomy has commonly been performed. However, osteoplastic surgery has been conducted in an increasing number of patients to reconstruct the posterior spinal element, which prevents postoperative subcutaneous fluid collection caused by cerebrospinal fluid leakage, postoperative wound infection caused by subcutaneous fluid collection and postoperative wound pain ${ }^{1,4,5,6,8,9,11,18)}$. For carrying out laminotomy, rotating high-power instruments such as high-speed drills has been used. These instruments work effectively in cutting bone; however, it has been indicated that bone cutting at a deep area in a narrow surgical field may result in dural and spinal injuries ${ }^{16)}$. In this report, to overcome these limitations and establish a safe and less invasive procedure, we performed laminoplastic laminotomy and hemilaminotomy using the scalpel-type ultrasonic bone curette. This instrument resects bones with oscillation without rolling movement 2,3,13,17). Because of its longitudinal and torsional oscillating motion, it facilitates bone resection during spinal surgery $7,12,14$ ). With the ultrasonic bone curette, bones are resected by ultrasonic oscillation, not by rotation movements, and the dura mater and nerve tissue absorb the oscillation ${ }^{10)}$. Thus, the risks of injuring the soft-tissue structures are supposed to be prevented using this instrument. There were no intraoperative spinal cord and root injuries in our 12 patients.

The ultrasonic bone curette has some technical advantages in cutting the laminae. This technique requires less expertise than that involved in the drilling technique. Because there is no rotational movement, the tip of the device is much more stable. As the width of bone resection is limited, $0.7 \mathrm{~mm}$, fixation of lamina with titanium plates is successful without leaving a wide space. The resected laminae were easily fused postoperatively in our 
cases. Furthermore, as the curette does not grab any cotton, bone cutting can be performed while protecting surrounding important neural and vascular structures with cottonoid patties. Concerning the hand pieces, they are light and can be readily manipulated with one hand ${ }^{14)}$. While the curette is being operated, the hand piece end is cooled by automatic irrigation of physiological saline. There is no danger of causing thermal injury to the surrounding important neural and vascular structures.

This scalpel-type ultrasonic bone curette is available only for cutting

bone. As the width of the tip is very thin, it is not used to remove osseous spurs or ossified lesions such as decompression of the nerve roots. However, there is another type of the tip, which is a curettes-type. The width of this curettes-type is $2.8 \mathrm{~mm}$. Use of the curettes-type ultrasonic bone curette is applicable to the nerve root decompressions during foraminotomy. We use this curettes-type ultrasonic bone curette in a deep area in a narrow surgical field such as lateral recess of the lumbar spine to decompress the nerve roots as foraminotomy. Using ultrasonic bone curette is safer than high-speed drills in such an operative field. However, as described in our cases, the dura mater which is calcified or compressed by the large tumor could not absorb the oscillation by the ultrasonic bone curette, which might cause dural tear. We postulate that no cerebrospinal fluid was available to provide a buffer between the dura mater and the tumor and that the dura mater did not absorb the oscillation of ultrasonic bone curette. It is then important to resect bone under an operating microscope visually confirming the tip end. In such cases, an initial drillbased rough bone cutting on the surface of the laminae followed by finer cutting with an ultrasonic bone curette would be advisable (Fig. 6). Especially in the large tumor located in lumbar lesion, it is difficult to check the tip of this device under an operating microscope without this surgical procedure. This procedure is a little time consuming comparing to the standard drilling, as this procedure consists of two steps. However, this two-step method contributes to safety cutting of the thick lamina. After removal of the cancellous bone by drilling as the first step, the ultrasonic bone curette is used to achieve finer cutting of the inner cortex of the lamina as the second step. Thus, this procedure allows confirming of the tip end in the relatively shallower operating field under an operating microscope. We can verify the feel of the solid sensation of the bone in the tip of this device during cutting the inner cortex so as not to injure the dural theca. We adopted this procedure since this case. There have been no dural tear using this surgical technique.

\section{Conclusion}

The scalpel-type ultrasonic bone curette is useful for cutting bone and effective for reconstruction of the laminae. Laminotomy with the scalpel-type ultrasonic bone curette is minimally invasive. We recommend using this instrument while visualizing the tip of the bone curette under an operating microscope especially in cases with calcified or tense dura by an 
intradural mass lesion.

\section{Disclaimer}

No authors have any financial ties to the manufacturer. 


\section{References}

1. Asazuma T, Nakamura M, Matsumoto M, et al. Postoperative changes of spinal curvature and range of motion in adult patients with cervical spinal cord tumors: analysis of 51 cases and review of the literature. $\quad J$ Spinal Disord Tech 17:178-182, 2004

2. Chang HS, Joko M, Song JS, et al. Ultrasonic bone curettage for optic canal unroofing and anterior clinoidectomy. Technical note. $\boldsymbol{J}$ Neurosurg 104:621-624, 2006

3. Hadeishi H, Suzuki A, Yasui N, et al. Anterior clinoidectomy and opening of the internal auditory canal using an ultrasonic bone curette. Neurosurgery 52:867-871, 2003

4. Hamburger C. T-laminoplasty - a surgical approach for cervical spondylotic myelopathy. Technical note. Neurochir (Wien) 132:131-133, 1995

5. Hara M, Takayasu M, Takagi T, et al. En Bloc laminoplasty performed with threadwire saw: Technical note. Neurosurgery 48:235-239, 2001

6. Herman JM, Sonntag V. Cervical corpectomy and plate fixation for postoperative kyphosis. $J$ Neurosurg 80:963-970, 1994

7. Hidaka K, Chiba Y, Takeda H. Clinical application of ultrasonic osteotome for the spinous process- splitting laminoplasty. Spinal Surg 12:19-24, 1998 (Jpn)

8. Hirabayashi K, Watanabe K, Wakao K. Expansive open-door laminoplasty for cervical stenotic myelopathy. Spine 8:693-699, 1983

9. Hirabayashi K, Satomi K. Operative procedure and results of expansive open-door laminoplasty. Spine 13:870-876, 1988

10. Howard BK, Beran SJ, Kenkel JM, et al. The effects of ultrasonic energy on peripheral nerves: implications for ultrasound-assisted liposuction. Plast Reconstr Surg 103:984-989. 1999

11. Itoh T, Tsuji H. Technical improvements and results of laminoplasty for compressive myelopathy in the cervical spine. Spine 10:729-736, 1985 
12. Kim K, Isu T, Matsumoto R, et al. Surgical pitfalls of an ultrasonic bone curette (SONOPET) in spinal surgery. Neurosurgery 59:390-393, 2006

13. Nagasawa S, Shimano H, Kuroiwa T. Ultrasonic aspirator with controllable suction system- variable action suction adapter and clinical experience with it. No Shinkei Geka 28:1083-1085, 2000 (Jpn)

14. Nakagawa H, Kim SD, Mizuno J, et al. Technical advantages of an ultrasonic bone curette in spinal surgery. $J$ Neurosurg Spine 2:431-435, 2005

15. Park AE, Heller JG. Cervical laminoplasty: use of a novel titanium plate to maintain canal expansion-- surgical technique. J Spinal Disord Tech 17(4):265-271, 2004

16. Satomi K, Ogawa J, Ishii Y, et al. Short-term complications and long-term results of expansive open-door laminoplasty for cervical stenotic myelopathy. Spine $J$ 1:26-30, 2001

17. Sawamura Y, Fukushima T, Terasaka S, et al. Development of a handpiece and probes for a microsurgical ultrasonic surgical aspirator: instrumentation and application. Neurosurgery 45:1192-1197, 1999

18. Steinmetz MP, Resnick DK. Cervical laminoplasty. Spine $J$ 6:274-281, 2006 


\section{Figure Legend}

Fig. 1.

Photographs of a scalpel-type ultrasonic bone curette, of which the tip is $0.7 \mathrm{~mm}$ wide: superior oblique view of the tip (upper), and lateral view of the tip (lower).

Fig. 2. Laminoplastic hemilaminotomy.

A: Intraoperative photograph of a scalpel-type ultrasonic bone curette-assisted cutting of the lamina. The tip is visible under an operative microscope.

B: Intraoperative photograph showing fixation of the lamina with miniplates for hemilaminotomy.

Fig. 3.

Schematic drawings of laminoplastic hemilaminotomy using a scalpel-type ultrasonic bone curette and titanium miniplates.

A: Unilateral separation of the paravertebral muscles.

B: Cutting of the lamina at the lamino-articular line and the base of the spinous process (preservation of the facet joint).

C: Intradural procedure (removal of the intradural tumor).

D: After dural suturing, resected laminae are fixed with miniplates.

Fig. 4. A 75-year-old woman with a cervical neurinoma

A: Preoperative cervical MR imaging showing a well-enhanced tumor in the $\mathrm{C} 7 / \mathrm{Th} 1$.

B: Postoperative cervical CT scan demonstrating sufficient reconstruction of the spinal canal.

C: Postoperative cervical CT scan taken 10 months after operation showing complete bony fusion of the lamina.

Fig. 5. A 38-year-old woman with a lumbar neurinoma

A, B, C: Preoperative lumbar MR imagings showing a well-enhanced large dumbbell-shaped tumor.

D, E: CT scans of the lumbar spine revealing a calcified dura mater at L1 to 3 level.

F : Postoperative cervical CT scan taken 11 months after operation showing bony fusion of the lamina. 
Fig. 6.

Schematic drawings of laminoplastic laminotomy in thick lamina.

A: At first, cutting of the surface of the laminae is roughly performed using a drill.

B: As a next step, an ultrasonic bone curette is used in the relatively shallower and wide operating field under confirmation of the tip. 
Table 1: Summary of cases of laminotomy performed with a ultrasonic bone curette

\begin{tabular}{|c|c|c|c|c|c|}
\hline $\begin{array}{c}\text { patient } \\
\text { No. }\end{array}$ & $\begin{array}{c}\text { age(y)/ } \\
\text { sex }\end{array}$ & pathology & $\begin{array}{c}\text { laminotomy } \\
\text { level }\end{array}$ & $\begin{array}{c}\text { No. of } \\
\text { laminotomy }\end{array}$ & $\begin{array}{c}\text { start of } \\
\text { fusion(months) }\end{array}$ \\
\hline 1 & $15 / \mathrm{w}$ & lipoma & S3-S4 & 2 & 6 \\
\hline 2 & $40 / \mathrm{m}$ & neurinoma & C2-C3 & 2 & 5 \\
\hline 3 & $27 / \mathrm{w}$ & neurinoma & C3-C4 & 2 & 6 \\
\hline 4 & $75 / \mathrm{w}$ & neurinoma & C7-T1 & 2 & 6 \\
\hline 5 & $53 / \mathrm{w}$ & neurinoma & T11-L1 & 3 & 7 \\
\hline 6 & $61 / \mathrm{w}$ & neurinoma & C2 & 1 & 5 \\
\hline 7 & $38 / \mathrm{w}$ & neurinoma & T12-L3 & 4 & 7 \\
\hline 8 & $64 / \mathrm{m}$ & cavernous & T11-T12 & 2 & 8 \\
\hline 9 & $23 / \mathrm{w}$ & perimedullary & C6-T2 & 4 & 10 \\
\hline 10 & $55 / \mathrm{m}$ & neurinoma & T11-T12 & 2 & 6 \\
\hline 11 & $64 / \mathrm{m}$ & lipoma & S1-S2 & 2 & 6 \\
\hline 12 & $73 / \mathrm{m}$ & neurinoma & T12-L3 & 4 & 6 \\
\hline average & 49.0 & & & 2.5 & 6.5 \\
\hline
\end{tabular}

All patients with at least a 6-month follow-up had a tendency of fusion of the resected laminae.

*AVF= arteriovenous fistula 

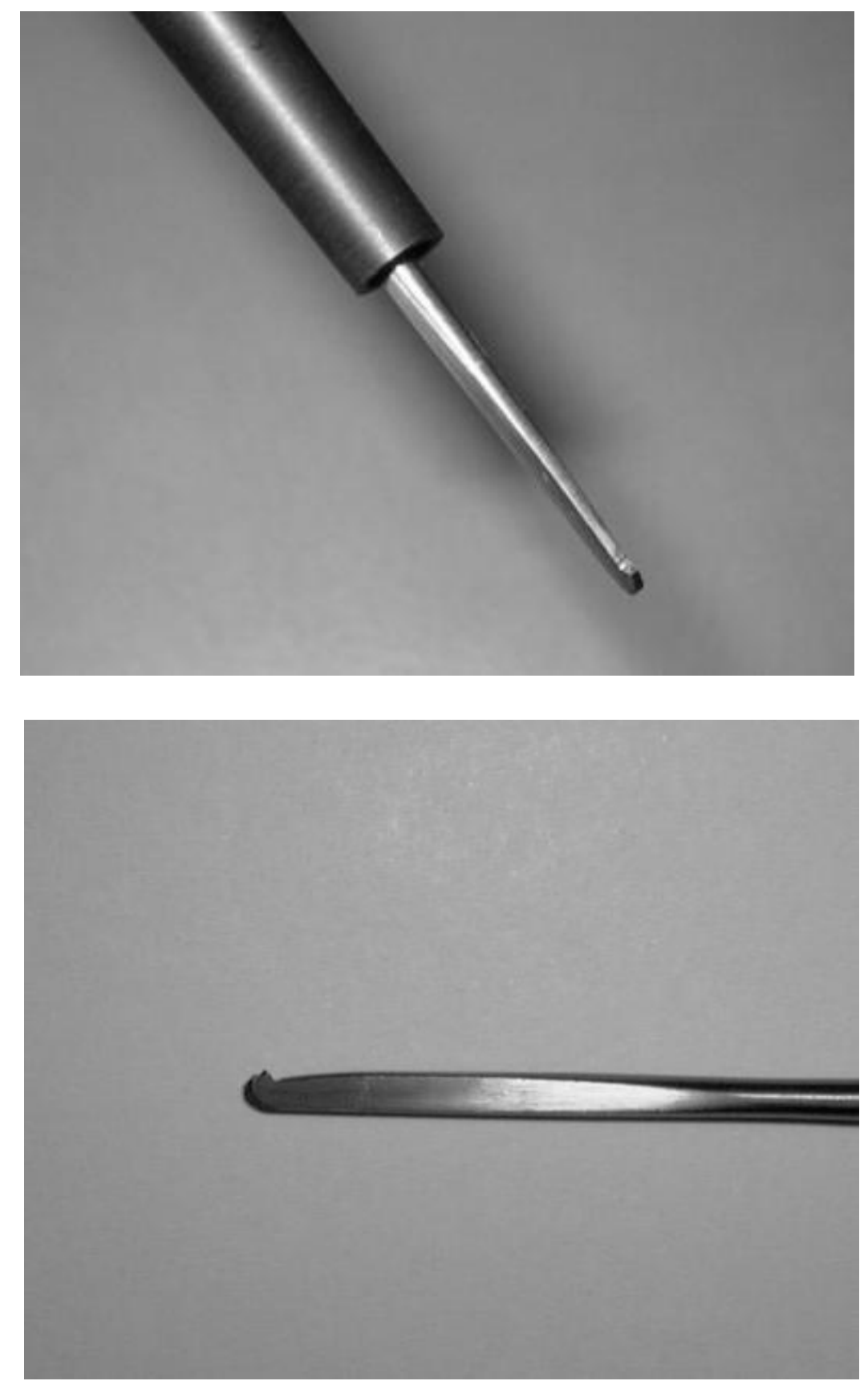

Fig. 1 


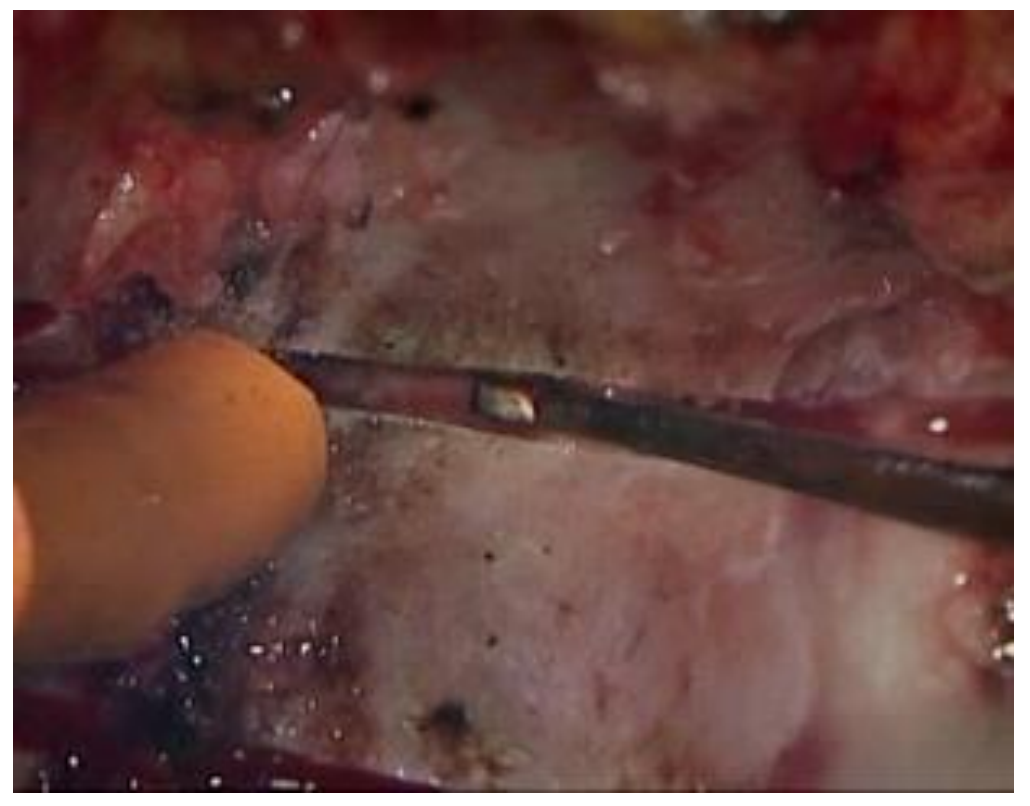

Fig. 2A

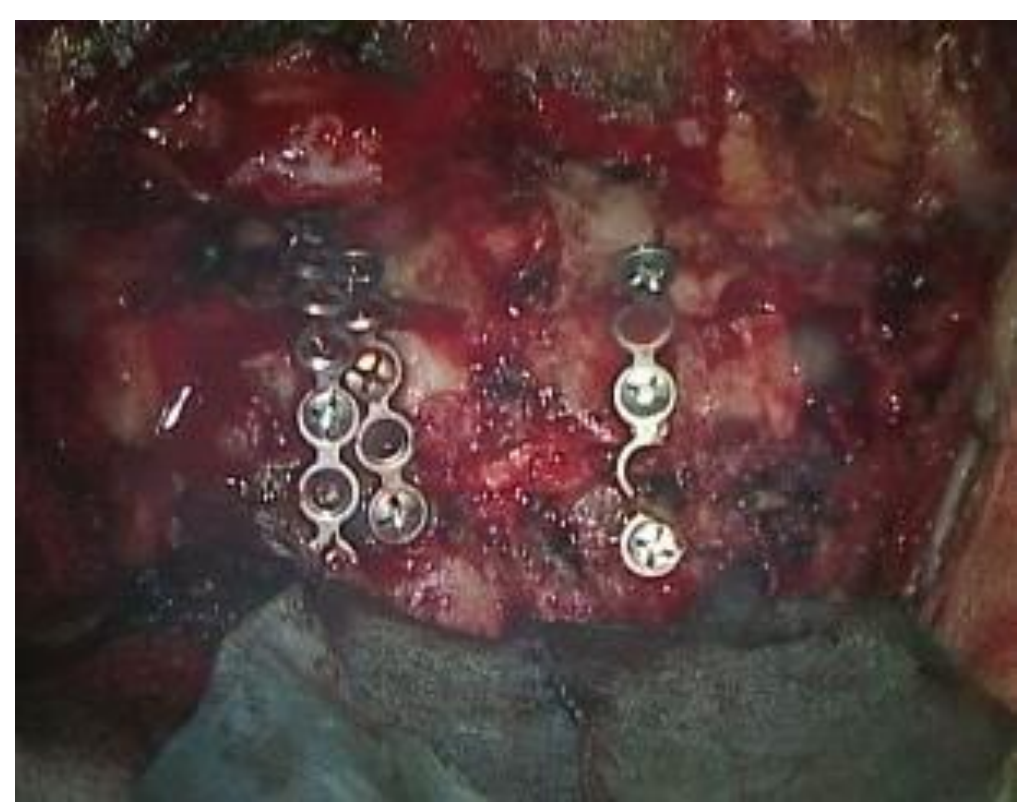

Fig. 2B 

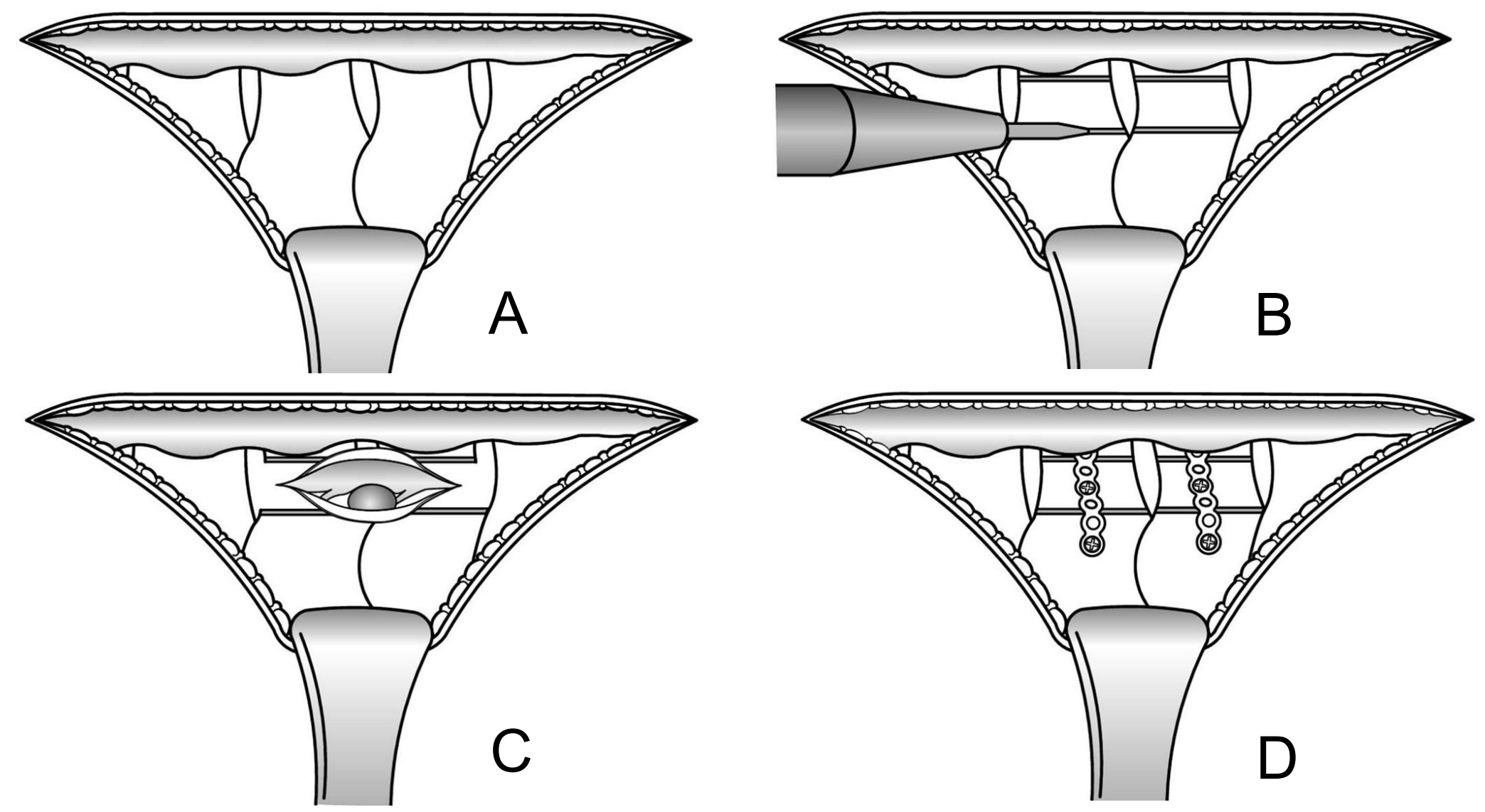

Fig. 3 


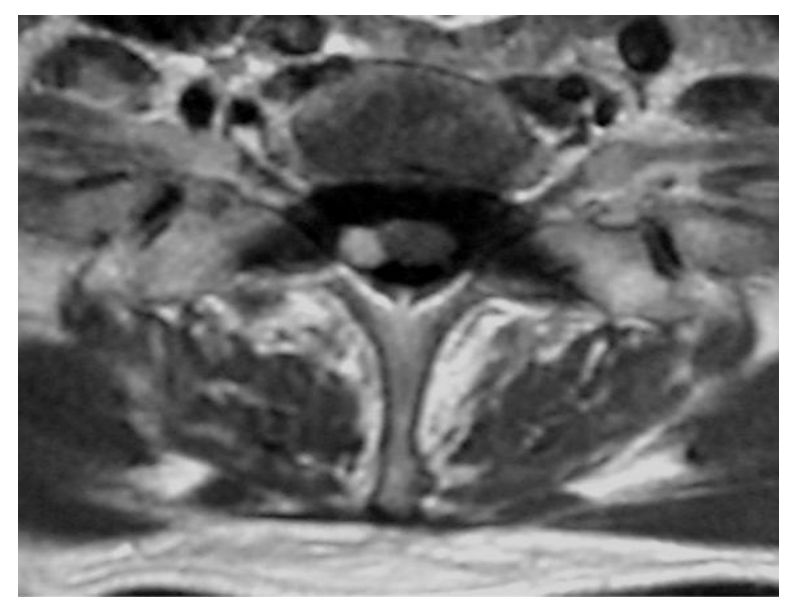

Fig. 4A
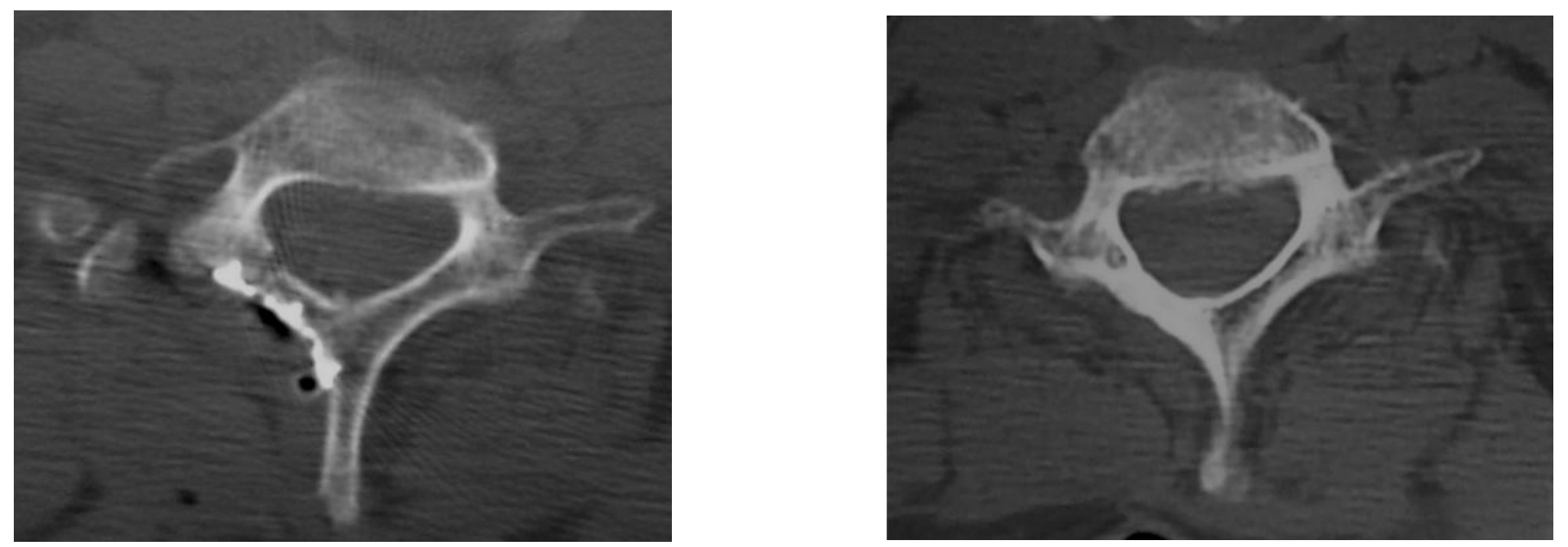

Fig. 4B

Fig. 4C 


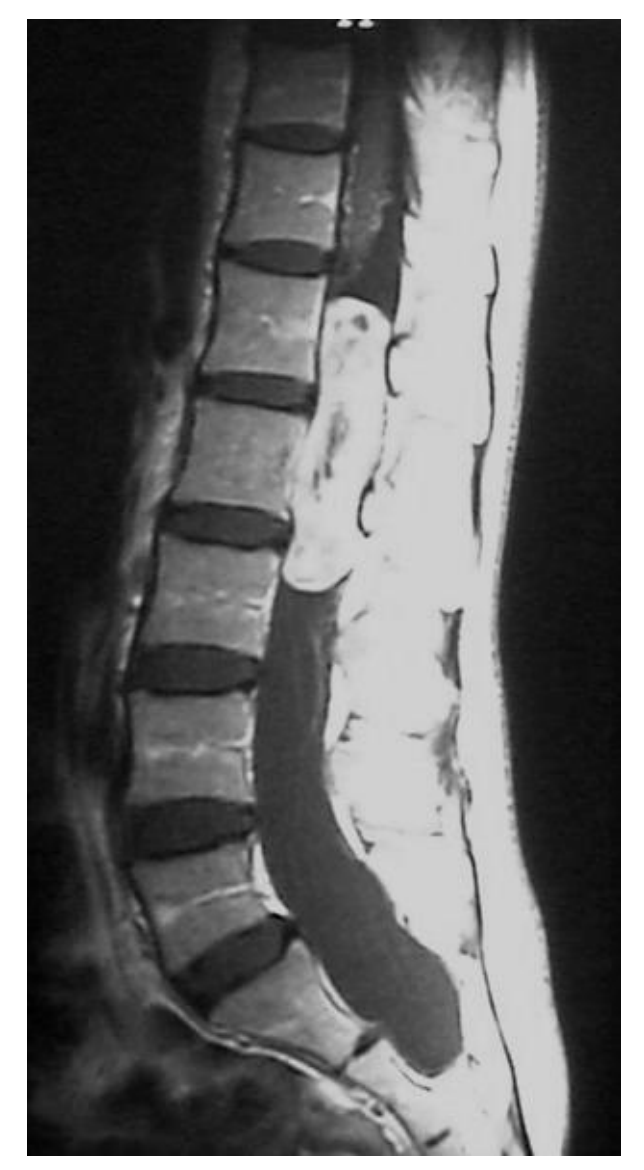

Fig. 5A

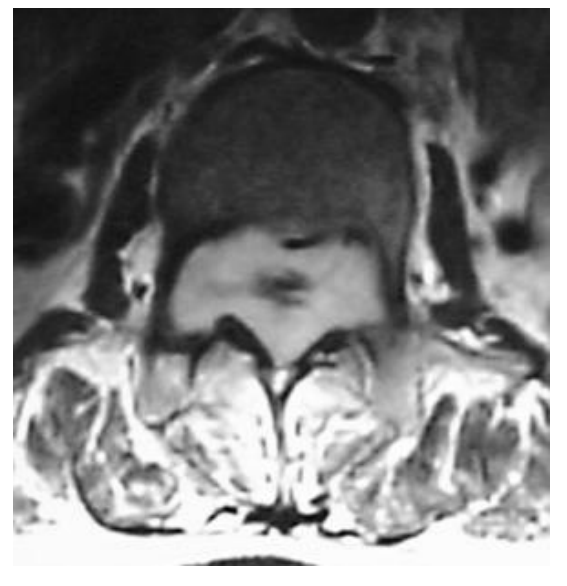

Fig. 5B

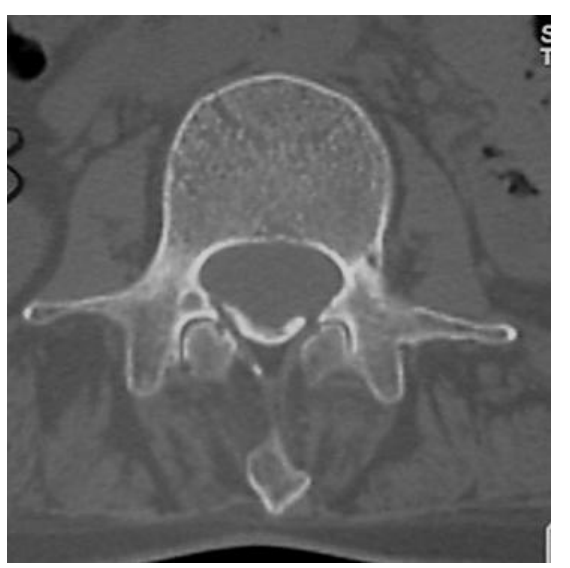

Fig. 5D

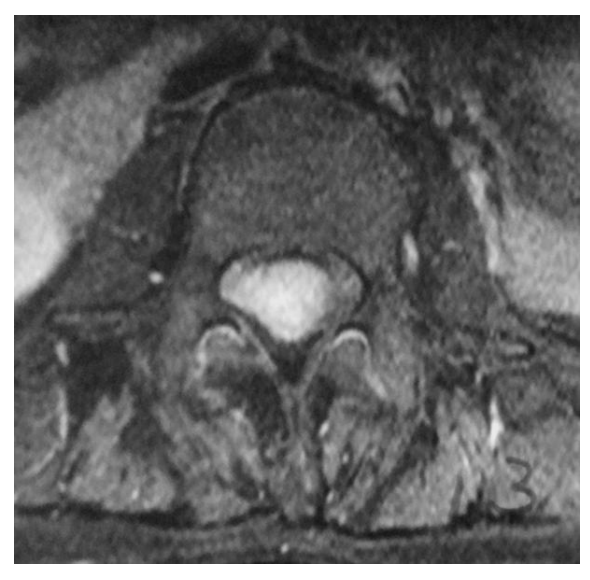

Fig. 5C

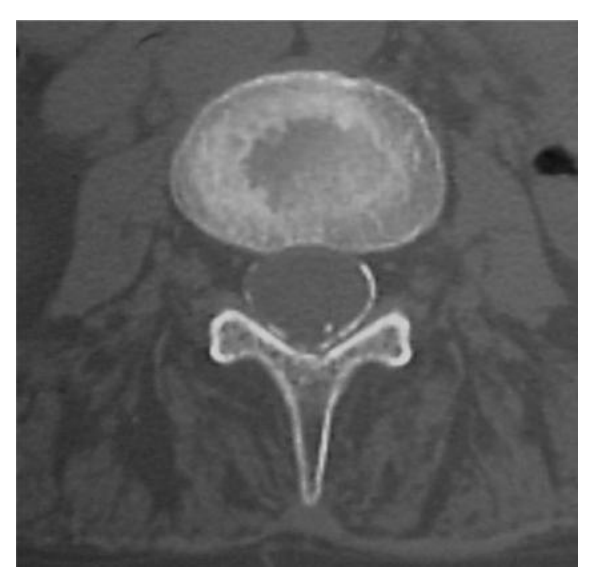

Fig. 5E

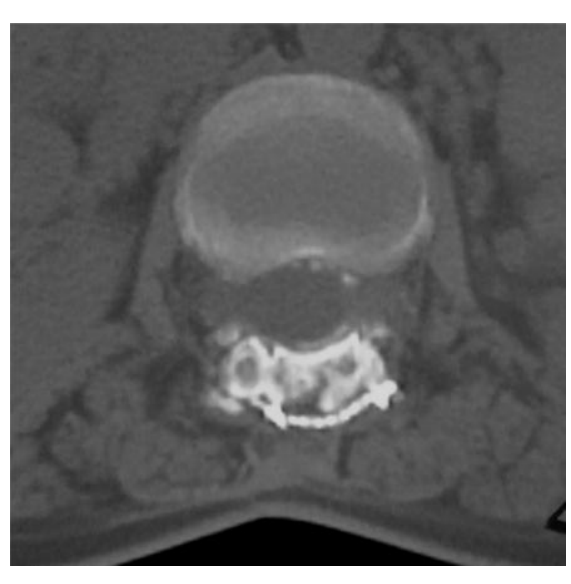

Fig. 5F 


$$
\text { of of }
$$

